stratification of individuals with pre-excitation. In addition, accessory pathway ablation did not eliminate the risk of SCD possibly due to multiple pathways. Finally, pre-excitation was associated with additional structural abnormalities in almost $50 \%$ of cases, underscoring the necessity of performing baseline echocardiography in all individuals and suggesting that the combination of pre-excitation with additional cardiac pathology may render individuals at higher risk of SCD.

\section{OBESITY AND SUDDEN DEATH. PATHOLOGICAL INSIGHTS FROM A LARGE PATHOLOGY REGISTRY}

${ }^{1}$ Gherardo Finocchiaro*, ${ }^{2}$ Michael Papadakis, ${ }^{2}$ Harshil Dhutia, ${ }^{1}$ Ahmed Merghani, ${ }^{1}$ Stathis Papatheodorou, ${ }^{2}$ Elijah Behr, ${ }^{2}$ Sanjay Sharma, ${ }^{1}$ Mary Sheppard. 'St George's University of London Hospital; ${ }^{2}$ St George's University Hospital; *Presenting Author

\subsection{6/heartjnl-2016-309890.67}

Aims Obesity is a rising public health problem and widely known risk factor for cardiovascular diseases. The relationship between obesity and sudden cardiac death (SCD) is unclear and based on small cohort studies. The aim of the study was to determine the main features and aetiologies in a large cohort of SCD occurred in obese subjects

Methods Between 1994 and 2014, 3684 consecutive cases of SCD were referred to our cardiac pathology centre. In 1954 body mass index (BMI) data were available; obesity was defined by a $\mathrm{BMI} \geq 30$. All subjects underwent macroscopic and microscopic post-mortem evaluation by an expert cardiac pathologist. Clinical information were obtained from the referring coroners.

Results Four hundred ninety-one patients (25\%) were obese. The average heart weight (HW) in obese patients was $505 \pm$ $170 \mathrm{~g}$ and $186(38 \%)$ had a HW of more than $500 \mathrm{~g}$. Obese patients were older at death $(39 \pm 14$ vs $35 \pm 16$ years in non-obese, $\mathrm{p}<0.001)$. In obese SCD victims the most common post-mortem findings were: normal heart (sudden arrhythmic death syndrome, SADS) $(\mathrm{n}=192,39 \%)$, followed by idiopathic left ventricular hypertrophy (ILVH) ( $\mathrm{n}=88$, $18 \%)$ and critical coronary artery disease (CAD) $(n=57$, $12 \%)$. Less frequently observed were hypertrophic cardiomyopathy $(\mathrm{HCM})(\mathrm{n}=24,4 \%)$ and arrhythmogenic right ventricular cardiomyopathy (ARVC) $(\mathrm{n}=22,4 \%)$. When compared with non-obese SCD victims, SADS was less common (39 vs 51\%, p < 0.001) while ILVH and critical CAD were more frequent (18 vs $3 \%, \mathrm{p}<0.001$ and 12 vs $6 \%, \mathrm{p}$ $<0.001$ respectively). In young patients $(<35$ years old) the prevalence of critical and non-critical CAD was significantly higher in obese subjects (23 vs $10 \%$ in non-obese, $\mathrm{p}<$ 0.001).

Conclusions Various conditionsunderlie SCD inobese patients, with a prevalence of SADS, ILVH and CAD. The degree of hypertrophy measured by heart weight appears in excess even after correction for body size, postulating its possible pathogenetic role in the development of fatal arrhythmias. Almost one in four young obese sudden death victims shows some degree of $\mathrm{CAD}$, underscoring the need for primary prevention in this particular subgroup.
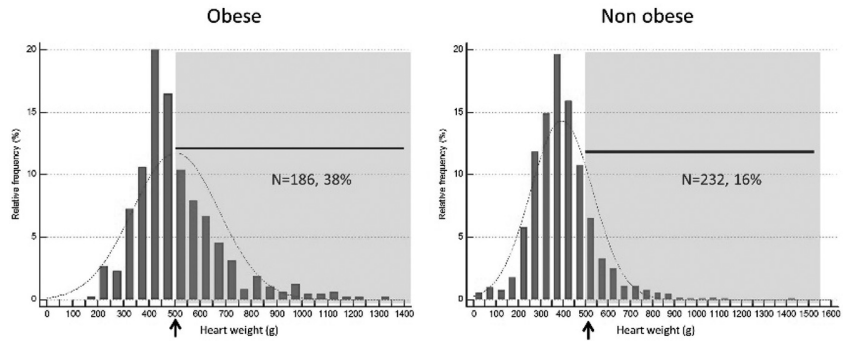

\section{Abstract 67 Figure 1}

\section{RIPPLE MAPPING THE VENTRICULAR SCAR: A NOVEL APPROACH TO SUBSTRATE ABLATION OF POST- INFARCT VENTRICULAR TACHYCARDIA TO PREVENT IMPLANTABLE DEFIBRILLATOR THERAPY}

${ }^{1}$ Vishal Luther*, ${ }^{2}$ Nicholas W Linton, ${ }^{2}$ Shahnaz Jamil-Copley, ${ }^{2}$ Michael Koa-Wing, ${ }^{2}$ Norman Qureshi, ${ }^{2}$ FuSiong Ng, ${ }^{2}$ Phang Boon Lim, ${ }^{2}$ Zachary Whinnett, ${ }^{2}$ David Wyn Davies, ${ }^{2}$ Nicholas S Peters, ${ }^{2}$ Prapa Kanagaratnam. ${ }^{1}$ North West Thames Deanery; ${ }^{2}$ Imperial College Healthcare NHS Trust; *Presenting Author

\subsection{6/heartjnl-2016-309890.68}

Introduction Ventricular Tachycardia (VT) is a common cause of mortality post myocardial infarction. Any mortality benefit of an implantable cardiac defibrillator (ICD) may be offset by the significant morbidity caused by VT shocks from the device. The VT circuit is dependent upon channels of surviving myocardium within the infarct substrate. As VT is often poorly tolerated, ablation of characteristic electrograms (fractionated and late potentials) associated with these channels in sinus rhythm is preferred to reduce ICD therapies, though recurrence rates remain high. Ripple Mapping (RM) displays all electrogram components from each anatomical point as a dynamic bar that protrudes from its 3D location. We have described how RM might visualize "conducting channels" (RM-CCs) of these characteristic electrograms within the infarcted substrate in a retrospective series. In this study, we used RM prospectively $\left(\mathrm{CARTO} 3 \mathrm{v}^{\mathrm{TM}}{ }^{\text {) }}\right.$ to characterize the VT substrate to guide ablation.

Methods Consecutive pts referred for VT ablation following episodes of sustained VT or ICD therapies post distant MI were included. High point density bipolar LV endocardial electrograms were collected in sinus rhythm or ventricular pacing within the infarct "scar" $(<1.5 \mathrm{mV})$ and reviewed for RMCC identification. Ablation targeted all RMCCs and isolated clusters of late activation considered an endocardial portion of an

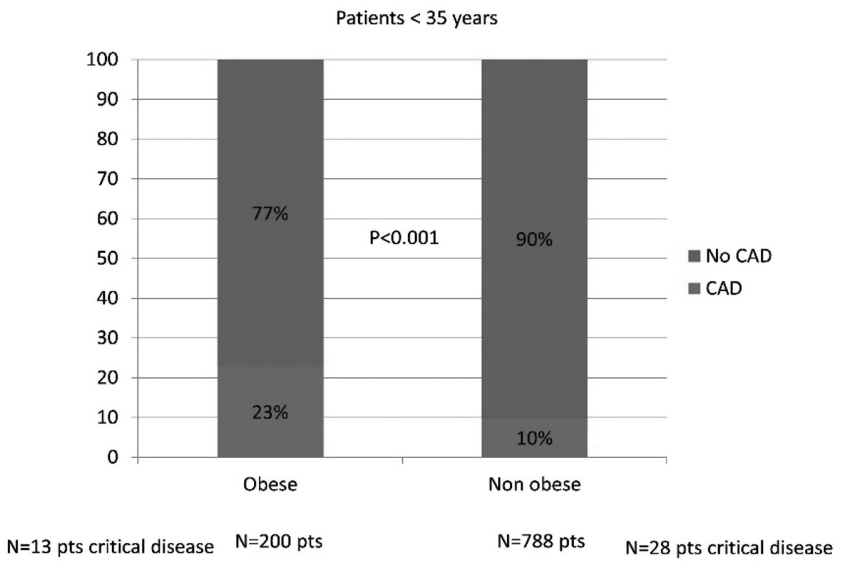

